\title{
The role of calcyclin gene in the proliferation and migration of gastric cancer cells
}

\author{
LIN ZHANG, YANHONG HOU, KAI WU and JUNSHAN ZHAI \\ Department of Gastroenterology, 309 Hospital of PLA, Beijing 100091, P.R. China
}

Received April 21, 2011; Accepted June 29, 2011

DOI: 10.3892/or.2011.1467

\begin{abstract}
The aim of this study was to investigate the influence of calcyclin on the growth, proliferation, apoptosis, invasion and cell cycle of gastric cancer cell lines in order to elucidate the role that calcyclin plays in gastric adenocarcinoma. Calcyclin cDNA was subcloned into a constitutive vector pcDNA3.1 followed by liposome-mediated transfection in the MKN45 gastric cancer cell line. Stable transfectants were selected and appraised. Specific inhibition of calcyclin was achieved using a vector-based siRNA system by transfection into the SGC7901 gastric cancer cell line. The apoptosis and cell cycle of these clones were analyzed by flow cytometry. Growth and proliferation were analyzed by cell growth curves and a colonyformation assay, respectively. The invasion of these clones was analyzed by a cell migration assay. MKN-calcyclin grew faster compared to MKN45 and MKN-PC (MKN45 transfected with pcDNA3.1 vector). SGC-SR1,2 grew slower compared to SGC7901 and SGC-SS1,2 (SGC7901 transfected with scrambled control duplexes). Cell cycle analysis showed that proportions of MKN-calcyclin and SGC-SR1,2 cells in G0/ G1 and G2/M were significantly different compared to those of their control groups, respectively $(\mathrm{P}<0.05)$. The apoptosis rate of $\mathrm{MKN}$-calcyclin was significantly lower compared to those of control groups $(\mathrm{P}<0.05)$. Results of colony-formation assay showed that the colony formation rate of $\mathrm{MKN}$-calcyclin was higher compared to those of control groups, otherwise the rates of SGC-SR1,2 were lower compared to those of their control groups $(\mathrm{P}<0.05)$. The results of the cell migration assay showed that the migration rate of $\mathrm{MKN}$-calcyclin was significant lower compared to those of its control groups, conversely, the migration rates of SGC-SR1,2 were significantly higher than those of their control groups $(\mathrm{P}<0.05)$. In conclusion, calcyclin can promote the growth and proliferation of gastric cancer cells and knockdown of calcyclin can restrain
\end{abstract}

Correspondence to: Dr Lin Zhang, Department of Gastroenterology, 309 Hospital of PLA, Beijing 100091, P.R. China

E-mail: stepinghuns@yahoo.com.cn

Key words: gastric cancer, calcyclin gene, cell apoptosis, cell cycle, RNAi the growth and proliferation of gastric cancer cells. It can help tumor cells maintain the malignant phenotype. However, it can depress the ability of invasion of gastric cancer cells, thus restraining the metastasis of gastric cancer.

\section{Introduction}

Gastric cancer is the second most common cause of death from cancer worldwide $(1,2)$ especially in many Asian countries, such as China, Japan and Korea. The incidence of gastric cancer varies substantially among racial and ethnic groups. The incidence is higher in eastern Asia than that in Western countries. Recently, major advances have been made in our understanding of the molecular and genetic alterations of this gastrointestinal malignancy. A number of these alterations, which are thought to contribute to carcinogenesis and cancer progression, have been investigated, such as those of oncogenes (k-ras, c-myc) (3-7), tumor suppressor genes (p53, retinoblastoma gene) $(8,9)$ and metastasis suppressor genes (KAI1 and nm23) (10,11). However, the more extensive genetic events that lead to the development of gastric cancer are not known. Understanding of this process may identify potential markers and therapeutic targets for gastric cancer.

The calcyclin gene is an important member of S100 family. The genes of this family are distributed in the region 1q211q22. A cluster of at least 16 types of S100 genes is located on 1q21. The S100 protein family constitutes a group of nearly 20 proteins that contain well-conserved EF-hand calcium binding domains (12-16).

Calcyclin is an EF-hand calcium binding protein $(17,18)$. Calcyclin cDNA was discovered by Hirschhorn et al (19) and further characterized by Calabretta et al (20). The protein was originally identified in Ehrlich ascites tumor cells by Filipek and Kuznicki (21) and Kuznicki et al (22). The properties and distribution of calcyclin are well established but its function is unclear. It has been suggested that calcyclin may be involved in cell proliferation, differentiation, and secretion $(23,24)$. In recent years, the up-regulation of calcyclin has been reported in a variety of tumors such as colorectal carcinoma, pancreatic carcinoma, hepatic carcinoma, breast carcinoma, melanoma and osteosarcoma (25-32) and is linked to metastasis. However, the exact intracellular roles of calcyclin in cancer have not been clarified. Yang et al (33) performed the serial analysis of gene expression (SAGE) experiment, microarrays and real-time polymerase chain reaction (RT-PCR) assays to 
compare the gene expression profiles between cancerous and adjacent tissues. They found that calcyclin was significantly up-regulated in gastric cancer tissue and thought that it was associated with gastric cancer tumorigenesis and assessment of calcyclin levels could be a promising tool for diagnosis of gastric cancer. On the other hand, Ning et al (34) prepared calcyclin-binding protein/Siah-1-interacting protein (CacyBP/ SIP) (calcyclin-binding protein which could interact with calcyclin) overexpressing and knockdown cell lines of gastric cancer. They found that CacyBP/SIP inhibited the proliferation of gastric cancer cells, suppressed tumorigenicity in vitro, and prolonged the survival time of tumor-bearing nude mice. Their results suggested that CacyBP/SIP could be a potential inhibitor of cell growth and invasion in the gastric cancer cell and it could be an antagonist to the role of calcyclin in gastric cancer. However, at present, the reports on the role of calcyclin in gastric cancer are limited.

Recently, using the technique of microarray analysis, we showed that the expression of calcyclin appeared to be up-regulated about 3 -fold in gastric cancer lesions relative to the expression by the peripheral normal gastric mucous membrane in our department $(35,36)$. Perhaps somewhat naively, we considered that such up-regulation of a putative carcinogenic gene was consistent with the possibility that greater proliferation, as well as enhanced invasive activity, would be more likely to occur in the cancer masses. Accordingly, we chose to examine the effects of up-regulation and down-regulation of calcyclin protein levels in cancer cells using the gene transfection and RNA interference (RNAi) approach, followed by assessment of the activity of treated cells in a range of proliferative or invasive assays. We report that calcyclin expression is crucial for tumor cell growth, survival and the maintenance of tumor cell parameters that may contribute to malignant potential. Our data suggest that calcyclin could partially be classified as a tumor-enhancing gene, but its role could be more complicated than what we think.

\section{Materials and methods}

Materials. The calcyclin monoclonal antibody was purchased from Abcam (Cambridge, MA, USA), Rabbit anti-mouse HPR (1:1,000; Dako, Copenhagen, Denmark) was used to recognize the corresponding proteins. pcDNA3.1 vector was prepared by our laboratory and common cell culture plates were purchased from Orange Co. (Braine-l'Alleud, Belgium). Transwell cell culture plates were $(8 \mu \mathrm{m}$ pore size; Transwell, BectonDickinson, Franklin Lakes, NJ, USA) purchased and prepared in our department. Annexin V-FITC apoptosis detection kit was purchased from Biosea Biotechnology Co., Ltd. (Beijing, China). All the primers used in this research were synthesized by Shanghai Boya Biotechnology Co., Ltd. (Beijing, China).

Cell lines and culture. The human gastric adenocarcinoma cell line (MKN45) was provided by the Shanghai Institute of Biotechnology and maintained by our department. The human gastric adenocarcinoma cell line (SGC7901) was obtained from Shanghai Cell Research Institute of Chinese Scientific Academy, stored and cultured in our laboratory. Dulbecco's modified Eagle's media (DMEM) containing $10 \%$ calf serum,
$100 \mathrm{IU} / \mathrm{ml}$ penicillin and $100 \mathrm{IU} / \mathrm{ml}$ streptomycin was used as the conventional culture medium. The culture procedures were performed under $37^{\circ} \mathrm{C}, 5 \% \mathrm{CO}_{2}$ and saturation humidity.

Investigation of calcyclin expression in SGC7901 and MKN45 cell lines. The expression of calcyclin in SGC7901 and MKN45 cell lines was detected to determine whether the cell lines could be used in the research. RT-PCR and immunocytochemistry were performed to detect the expression of calcyclin in cells.

Transfections. Human gastric cancer MKN45 cells were transfected with cDNA of the calcyclin gene. For the stable transfections, cDNA was inserted into the pcDNA3.1 expression vector (Invitrogen, San Diego, CA, USA) between the $E c o$ RI and BamHI site. The orientation of the insert was confirmed by restriction digestion and DNA sequencing. The pcDNA3.1 vector inserted with the calcyclin cDNA was renamed as pcDNA3/ calcyclin and was transfected into MKN45 cells using a Lipofectamine 2000 liposome transfection kit, (Boehringer Mannheim, Indianapolis, IN, USA). The pcDNA3.1 empty vector transfection group and the untreated control group were established. Putative transfectants were then selected by antibiotic resistance in cell culture medium containing $800 \mu \mathrm{g} / \mathrm{ml} \mathrm{G} 418$. After 7 weeks of culture in the presence of G418, the remaining cells were isolated with cloning cylinders and transferred into 24-well dishes. Eight and thirteen positive clones were respectively obtained in the pcDNA3/calcyclin vector transfection group (MKN-calcyclin) and pcDNA3.1 empty vector transfection group (MKN-PC). These selected clones were taken for identification and frozen for future use.

Calcyclin small interfering RNA (siRNA) synthesis and manipulation. We obtained the whole cDNA sequence of calcyclin from GenBank and used OligoEngine software to select two targeted fragments for RNAi as follows: 1, aaggagctcaccatt ggctcg (390-410), 2, aagcacacctgagcaagaag (432-452). These 21 nucleotide DNA sequences corresponded to calcyclin coding nucleotides.

To control for the specificity of the RNAi effect, an independent DNA duplex was designed and produced respectively with 1 point mutation compared with the calcyclin siRNA duplex; this served as the control for the transfection procedure. The scrambled control sequence was as follows: 1 , aaggagatcaccattggctcg; 2, aagcacacactgagcaagaag. A BLAST search of the calcyclin siRNA against the GenBank _ EMBL _ DDBJ _ PDB sequences was performed and the results identified only one gene. Human mRNA encoding the calcyclin was targeted. BLAST searches of the control siRNA against the same sequences showed no other human gene to be targeted. According to the selected targeted fragments, two calcyclin siRNA duplexes and two scrambled control duplexes were synthesized as follows: S1, 5'-cgacggagctcaccattggctcg gagtactgcgagccaatggtgagctccttttt-3' and 3'-gcctcgagtggtaacc gagcctcatgacgctcggttaccactcgaggaaaaagatc-5'; S2, 5'-cgacgcac accctgagcaagaaggagtactgcttcttgctcagggtgtgcttttt-3' and 3 '-gc gtgtgggactcgttcttcctcatgacgaagaacgagtcccacacgaaaaagatc-5'.

The scrambled control duplexes were as follows: S1s, 5'-cga cggagatcaccattggctcggagtactgcgagccaatggtgagctccttttt- $3^{\prime}$ and 3'-gcctctagtggtaaccgagcctcatgacgctcggttaccactcgaggaaaaagatc-5'; 
S2s, 5'-cgacgcacacactgagcaagaaggagtactgcttcttgctcagggtgtg cttttt-3' and 3'-gcgtgtgtgactcgttcttcctcatgacgaagaacgagtcccac acgaaaaagatc-5'.

All the DNA fragments were chemically synthesized, purified and annealed by Dharmacon Research (Lafayette, $\mathrm{CO}$, USA) and manipulated according to the manufacturer's instructions. These duplex DNA fragments were inserted into IMG-800 RNAi vector (Imgenex, San Diego, CA, USA) between the $X b a \mathrm{I}$ and $S c a \mathrm{I}$ site. The orientation of the insert was confirmed by restriction digestion and DNA sequencing, and then vector-based siRNAs were amplified.

Cell culture and RNA transfection. SGC7901 cells were grown in DMEM medium (Invitrogen, Paisley, UK) supplemented with $10 \%$ fetal calf serum (FCS) without antibiotics.

Twenty-four hours before transfection, $1 \times 10^{6}$ cells were seeded into a T75 flask in $15 \mathrm{ml}$ DMEM medium with $10 \%$ FCS. The Lipofectamine 2000 liposome transfection kit, (Boehringer Mannheim) was used to transfect vector-based siRNAs into cells according to the procedures recommended by the manufacturer. An amount of 1 nmole siRNA duplex was used for each T75 flask. In cases of apparent overconfluence, assessed by simple visual examination, cells were split on the second day after the transfection but the cells were maintained in various media containing the siRNA duplexes at the appropriate concentration. All assays were carried out on Day 5 after transfection, except the growth curve study, which was initiated on Day 2 after the transfection and continued over the subsequent 7 days. Two groups of SGC7901 cells transfected with two siRNA duplexes were renamed as SGC-SR1 and SGC-SR2. Two other groups of SGC7901 cells transfected with scrambled control duplexes were renamed as SGC-SS1 and SGC-SS2 respectively. On the fifth day after transfection, the effect of siRNA on the gene expression was analyzed.

Analysis of transfectants. RT-PCR and Western blot analysis were respectively performed to detect the mRNA and protein levels of calcyclin in each sample, and immunocytochemical analysis was used to detect the expression of calcyclin protein in the MKN-calcyclin, MKN-PC, SGC-SR1,2, SGC-SS1,2 cells in situ. Densitometry of the RT-PCR and Western blot analysis results of the SGC-SR1,2, SGC-SS1,2 and SGC7901 groups was performed, and the data represent average values from 5 replicates respectively. Expression of calcyclin mRNA and protein from the cells of each group was normalized to the $\beta$-actin band and compared with controls. Bands were detected by chemiluminescence and quantified using NIH Image (v1.60). Data were expressed as a percentage of control, and were analyzed using the Student's t-test, with $\mathrm{P}<0.05$ being considered significant.

Cell growth curves. All of $8 \mathrm{MKN}$-calcyclin cell clones, 13 MKN-PC cell clones and untreated MKN45 cells were used. The cells of each clone were inoculated into 24-well culture plates at the concentration of $5 \times 10^{4} / \mathrm{ml}$. After the cells were plated, they were washed once with phosphate buffer solution (PBS) and then trypsinized in $0.5 \mathrm{ml}$ of Trypsin/EDTA (ethylenediaminetetraacetic acid) and counted in triplicate at 1 to 7 days using a cell counter (Beckman Coulter, Inc., Fullerton, CA, USA). The mean values of all $8 \mathrm{MKN}$-calcyclin cell clones and $13 \mathrm{MKN}-\mathrm{PC}$ clones at different time points were calculated, and growth curves were plotted.

Two days after the siRNA transfection, cells of SGC-SR1,2, SGC-SS1,2 and untreated SGC7901 groups were trypsinized and seeded at $2 \times 10^{4} /$ well in 6 -well plates. Subsequently, cells were trypsinized and counted each day after plating.

Colony formation assay. Each clone of MKN-calcyclin, MKN-PC, and untreated MKN45 groups was examined. One thousand cells of each clone were respectively seeded in a $9 \mathrm{~cm}$ cell culture dish. After 18 days of culture in DMEM containing FCS, cell colonies were fixed and stained with $0.5 \%$ methylene blue (Sigma, Poole, Dorset, UK) in ethanol and the number of cell colonies with more than 50 cells was counted in each dish (colony formation rate $=$ number of colonies in each dish $/ 1,000$ ). Three reduplicate dishes were used from each clone.

On the fifth day after transfection, 1,000 cells from each sample of SGC-SR1,2, SGC-SS1,2 and untreated SGC7901 groups were seeded in 6-well plates and kept growing in $4 \mathrm{ml}$ DMEM with $10 \%$ FCS. After 16 days culture, cell colonies were fixed and stained with $0.5 \%$ methylene blue (Sigma) in ethanol. All colonies visible by eye were counted separately for each sample and their colony formation rates were evaluated.

Analysis of cell cycle and apoptosis. All cell clones of the MKN-calcyclin, MKN-PC, and untreated MKN45 groups and all cell samples of the SGC-SR1,2, SGC-SS1,2 and untreated SGC7901 groups were detected by flow cytometry for analysis of the cell cycle and apoptosis. When the cells covered $70 \%$ of the area of the cell culture plates, the serumfree culture medium was used for synchronization. After $24 \mathrm{~h}$ of continuous culture, the cells were harvested and fixed by $100 \%$ ethanol, then prepared for single cell suspensions. After DNA staining, the cell cycles of the samples were measured on a FACScalibur cytometer (Becton-Dickinson). Analysis was performed with the CellQuest software. After synchronization and $24 \mathrm{~h}$ continuous culture, the cells were harvested and fixed. Propidium iodide (PI) and Annexin V-FITC double staining was performed, and flow cytometry was used to detect cell apoptosis. Three replicate tests on every sample were performed in each group, the average values of these groups were calculated respectively, and comparison between these groups was conducted.

Analysis of the influence of specific down-regulation of calcyclin on cellular proliferation by PCNA staining. The cells of the SGC-SR1,2, SGC-SS1,2 and untreated SGC7901 groups were immunostained for proliferating cell nuclear antigen (PCNA) to assess the activity of cellular proliferation. Cultured cells were fixed with $4 \%$ paraformaldehyde (PFA) in PBS for $1 \mathrm{~h}$, and to block non-specific staining, they were treated with $2 \%$ bovine serum albumin (BSA) and $0.1 \%$ Triton X-100 in PBS for $1 \mathrm{~h}$ at room temperature. After treatment with $3 \% \mathrm{H}_{2} \mathrm{O}_{2}$ for $10 \mathrm{~min}$ to block endogenous peroxidase activity, primary mouse antibody raised against PCNA (Clone PC10; Dako, Carpinteria, CA, USA) was applied for $1 \mathrm{~h}$, the secondary biotin-labelled goat anti-mouse antibody was added for $10 \mathrm{~min}$; and the avidin-biotin peroxidase complex (ABC) (Dako) was applied for $10 \mathrm{~min}$. 3,3'-Diaminobenzidine (DAB) was used as the chromogen. Finally, the cells were counterstained with 


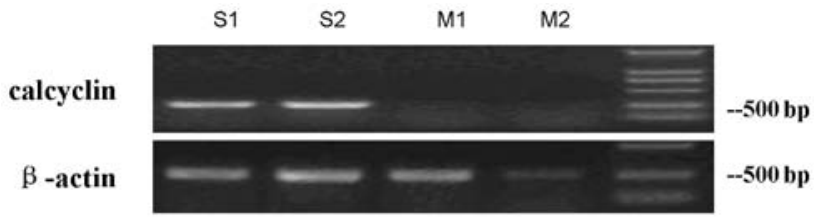

Figure 1. The results of RT-PCR for calcyclin in SGC7901 and MKN45 cells. S1, S2 were the results of RT-PCR for calcyclin and $\beta$-actin in SGC7901 cells, M1, M2 were the results of RT-PCR for calcyclin and $\beta$-actin in MKN45 cells. The calcyclin and the $\beta$-actin fragments were about $500 \mathrm{bp}$. The results showed that calcyclin expression was present in SGC7901 cells, but not detectable in MKN45 cells.

Harris's hematoxylin for 3 min and coverslips applied with a xylene-based mounting medium. For the quantification of PCNA immunostaining, 15 fields were analyzed per sample. The PCNA-labeling index (PCNA positive proportion) was expressed as the percentage of the number of PCNA-labelled nuclei divided by the total number of nuclei examined under a microscope (20x objective) and the average value of each group was calculated. The PCNA positive proportions were compared between SGC-SR1,2 and their control groups.

Cell migration assay. The cell migration assay was performed using FCS-coated polycarbonate filters $(8 \mu \mathrm{m}$ pore size; Transwell, Becton-Dickinson). The membrane undersurface was coated with $200 \mu \mathrm{FCS}$ for $1 \mathrm{~h}$ at $37^{\circ} \mathrm{C}$ and blocked with $200 \mu 1$ migration buffer (0.5\% BSA in DMEM) for $30 \mathrm{~min}$ at $37^{\circ} \mathrm{C}$. The lower chamber was filled with $500 \mu$ l of migration buffer, following which cells were plated in the upper chamber of 4 wells/treatment at a density of $1 \times 10^{5}$ in $100 \mu \mathrm{l}$ of migration buffer and incubated at $37^{\circ} \mathrm{C}$ for $4 \mathrm{~h}$. Following incubation, cells in the upper compartment were trypsinized and counted by the CASY 1 counter (Sharfe System, Reutingen, Germany). Cells that had migrated to the lower surface of the filter were also trypsinized and counted. The migration rate was obtained by dividing the cell number in the lower chamber by the sum of the cell number found in both the lower chamber and the upper chamber.

\section{Results}

Expression of calcyclin gene in SGC7901 and MKN45 cell lines. The expression of the calcyclin in the gastric adenocarcinoma cell strains SGC7901 and MKN45 was detected by RT-PCR and immunocytochemical analysis. All the results in the MKN45 cell strain were negative, which indicated that there was no detectable expression of calcyclin gene or protein in untreated MKN45 cells. On the other hand, the results in the SGC7901 cell strain were positive. The results of the immunocytochemical assay showed that the expression of calcyclin in SGC7901 cells was mainly distributed in the cytoplasm and membrane but there was no obvious positive signal in the cell nucleus (Figs. 1 and 2).

Expression of calcyclin gene in transfectants. The results of RT-PCR, Western blotting and immunocytochemical analysis showed that the expression of calcyclin significantly increased in MKN-calcyclin cells when compared with the untreated MKN45 cells or MKN-PC cells. The results of the immuno-

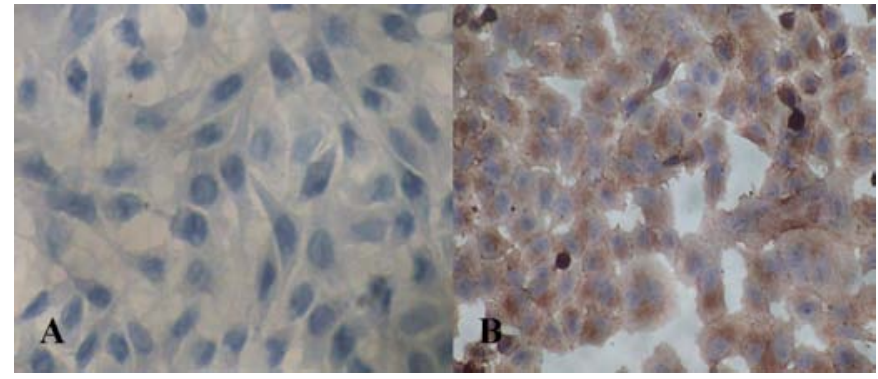

Figure 2. Immunocytochemistry of calcyclin in the MKN45 and SGC7901 cell lines (x200). (A) The results showed that there was no detectable expression of calcyclin in MKN45 cells. (B) The brown positive signals were mainly distributed in the cytoplasm and membrane. The results showed that calcyclin expression was detectable in the SGC7901 cell line.

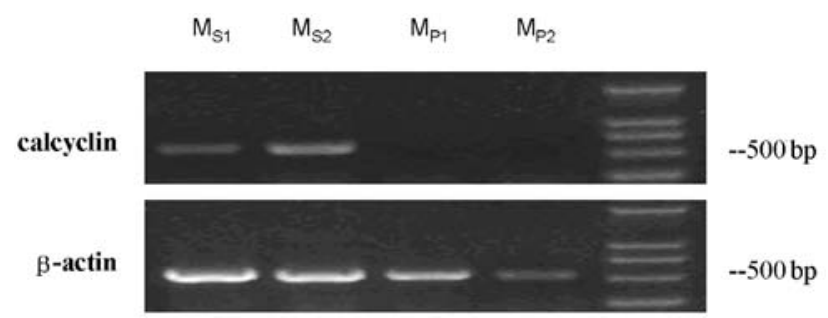

Figure 3. RT-PCR of calcyclin in MKN-calcyclin and MKN-PC cells. $\mathrm{M}_{\mathrm{S}}$ and $\mathrm{M}_{\mathrm{S} 2}$ denote the results of RT-PCR for calcyclin and $\beta$-actin control in MKN-calcyclin cells, $\mathrm{M}_{\mathrm{P} 1}$ and $\mathrm{M}_{\mathrm{P} 2}$ indicate the results of RT-PCR for calcyclin and $\beta$-actin control in MKN-PC cells. The results showed that expression of calcyclin gene was detectable in MKN-calcyclin cells but not in MKN-PC cells.

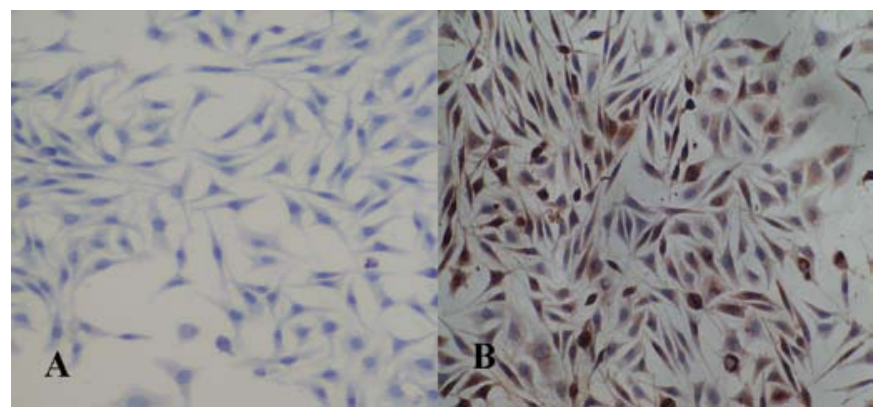

Figure 4. Immunocytochemistry of calcyclin in MKN-PC and MKNcalcyclin cells (x200). (A) There was no positive signal in MKN-PC cell. (B) The brown positive signals were mainly distributed in cytoplasm of MKNcalcyclin cells. The results showed that there was expression of calcyclin in the MKN-calcyclin cell line but not in the MKN-PC cell line.

cytochemical assay showed that the expression of calcyclin in MKN-calcyclin cells was mainly distributed in the cytoplasm and there was no obvious positive signal in the cell nucleus and membrane (Figs. 3-5). The results of RT-PCR, Western blotting and immunocytochemical assay showed that calcyclin siRNA can efficiently and specifically decrease endogenous calcyclin expression. Only the calcyclin siRNA caused the down-regulation of calcyclin protein, while calcyclin protein levels in the scrambled siRNA and untreated control samples showed no change (Figs. 6-8). These results indicate that only specific siRNA caused complementary mRNA degradation and thus the decrease of the corresponding protein. Calcyclin 


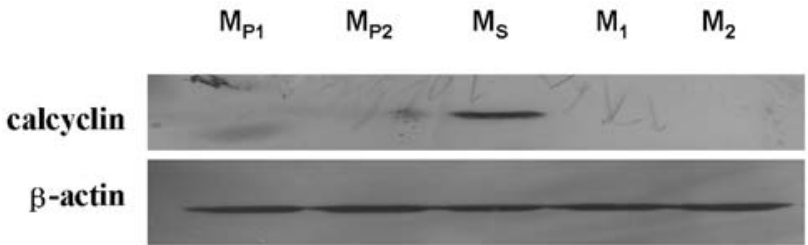

Figure 5. Western blotting of calcyclin in MKN-calcyclin, MKN-PC and MKN45 cells. $\mathrm{M}_{\mathrm{S}}$ indicates the levels of calcyclin and $\beta$-actin control in MKNcalcyclin cells, $\mathrm{M}_{\mathrm{P} 1}$ and $\mathrm{M}_{\mathrm{P} 2}$ indicate the calcyclin and $\beta$-actin control levels in MKN-PC cells, $M_{1}$ and $M_{2}$ those in MKN45 cells. The results show that calcyclin is expressed in MKN-calcyclin cells but not in MKN-PC or MKN45 cells.

A

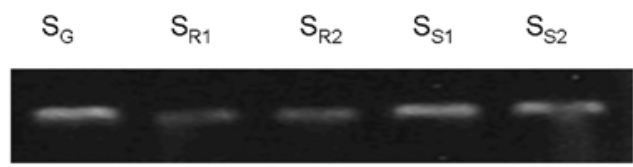

$\beta$-actin

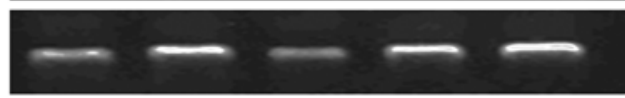

B

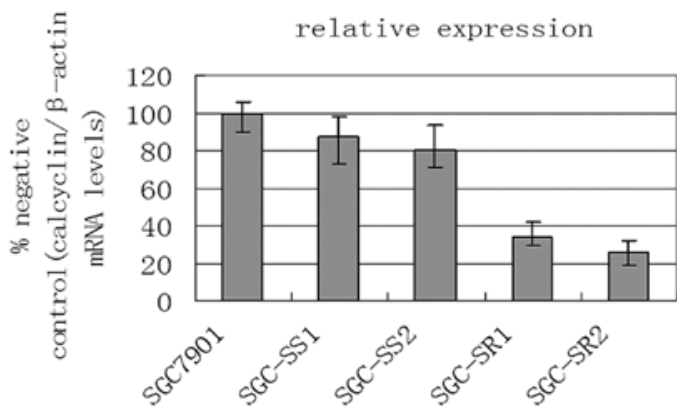

Figure 6. RT-PCR of calcyclin in SGC-SR1, SGC-SR2, SGC-SS1, SGC-SS2 and untreated SGC7901 cells. (A) $\mathrm{S}_{\mathrm{S} 1}$ and $\mathrm{S}_{\mathrm{S} 2}$ indicate the results of RT-PCR for calcyclin and $\beta$-actin control in SGC-SS1 and SGC-SS2 cells, respectively, and $S_{R 1}$ and $S_{R 2}$ indicate those in SGC-SR1 and SGC-SR2 cells, respectively. $\mathrm{S}_{\mathrm{G}}$ indicates the results in untreated SGC7901 cell. The results show that the expression levels of the calcyclin gene in SGC-SR 1 and SGC-SR2 cells were decreased when compared with the control groups. (B) Levels of calcyclin and $\beta$-actin mRNA of cells were detected by RT-PCR 5 days after siRNA transfection. About 20-30\% relative expression rates in SGC-SR1 and SGCSR2 groups could be detected when compared with untreated SGC7901.

mRNA and protein were decreased to about 30 or $20 \%$ of the control level respectively by the fifth day after the calcyclin siRNA transfection (Figs. 6-8).

The influence of calcyclin gene on cell growth. The results of the cell growth curve assay showed that MKN-calcyclin cells grew significantly faster than MKN-PC and untreated MKN45 cells, respectively $(\mathrm{P}<0.05)$, and there was no significant difference between the control groups. At Day 4, 5, 6 and 7 after inoculation, the average cell counts of the MKN-calcyclin group were $2.86 \times 10^{5}, 3.42 \times 10^{5}, 4.27 \times 10^{5}$ and $4.64 \times 10^{5}$ respectively, which were significantly higher than those of the two control groups $(\mathrm{P}<0.05)$ (Fig. 9A).

The growth curves of SGC-SR1, SGC-SR2, SGC-SS1, SGC-SS2 and untreated SGC7901 (Fig. 9B) groups showed that treatment with calcyclin siRNA, but not with scrambled siRNA, could restrain cell growth over a period of 7 days. At Day 5, 6 and 7 after inoculation, the average cell counts of the SGC-SR1 group were $0.96 \times 10^{5}, 1.26 \times 10^{5}$ and $1.56 \times 10^{5}$ respec-

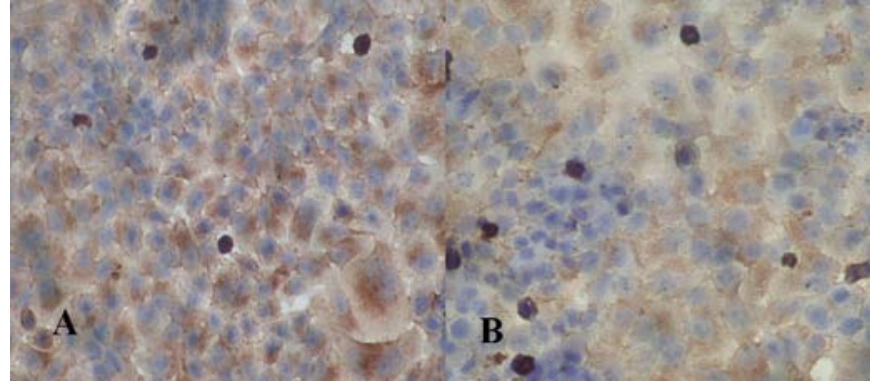

Figure 7. Immunocytochemistry of calcyclin in SGC-SS1 and SGC-SR1 cell lines (x200). (A) The brown positive signals were mainly distributed in the cytoplasm and membrane of SGC-SS1 cells. (B) The brown positive signals were mainly distributed in the cytoplasm of SGC-SR1 cells and the intensity of positive signals decreased when compared with those of SGC-SS1 cells The results showed that the expression level of calcyclin in the SGC-SR1 cell line was reduced.

A

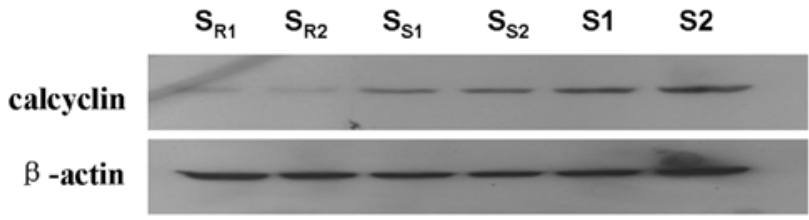

B

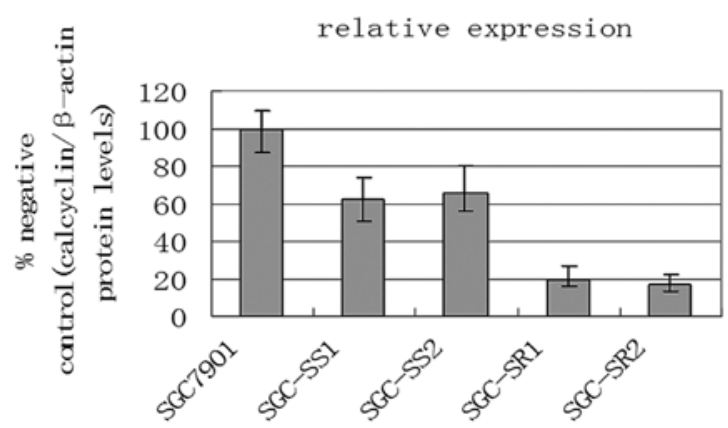

Figure 8. Western blotting of calcyclin in SGC-SR1, SGC-SR2, SGC-SS1, SGC-SS2 and untreated SGC7901 cells. (A) $\mathrm{S}_{\mathrm{R} 1}$ and $\mathrm{S}_{\mathrm{R} 2}$ indicate the results of Western blotting for calcyclin and $\beta$-actin control in the SGC-SR1 and SGC$\mathrm{SR} 2$ cells, respectively and $\mathrm{S}_{\mathrm{S} 1}$ and $\mathrm{S}_{\mathrm{S} 2}$ those in SGC-SS1 and SGC-SS2 cells, respectively. S1 and S2 indicate the results in the untreated SGC7901 cell line. The results showed that the expression levels of calcyclin in SGC-SR1 and SGC-SR2 cell lines were reduced when compared with the control groups. (B) Expression levels of calcyclin and $\beta$-actin proteins of cells detected by Western blot analyses 5 days after siRNA transfection. About $15-20 \%$ relative expression rates in SGC-SR1 and SGC-SR2 groups could be detected when compared with the untreated SGC7901 cells.

tively, which resembled those of the SGC-SR2 group and were significantly lower than those of the control groups $(\mathrm{P}<0.05)$.

The influence of calcyclin gene on colony formation. The colony formation rate of MKN-calcyclin cells $(0.57 \pm 0.05)$ was significantly higher than that of their control groups $(\mathrm{P}<0.05)$. There was no significant difference between these control groups ( $\mathrm{P}>0.05)$ (Fig. 10A). It is apparent that transfection with calcyclin siRNA increased the capacity of these cells to establish colonies to a highly significant degree.

The examples of data from a colony formation assay for SGC-SR1,2 cells are shown in Fig. 10B which were significantly lower than those of their respective control groups 
A

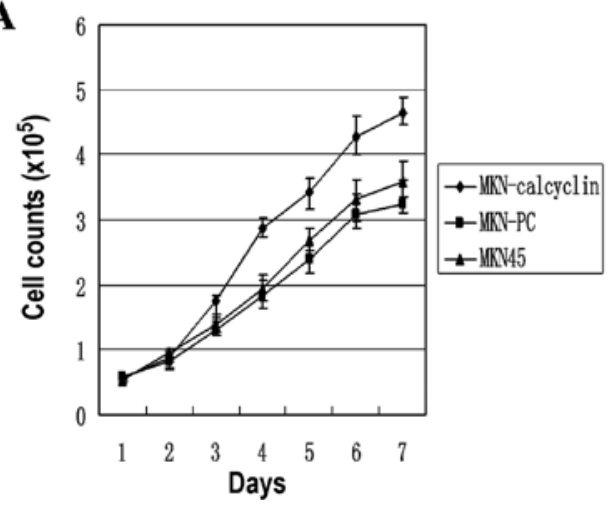

B

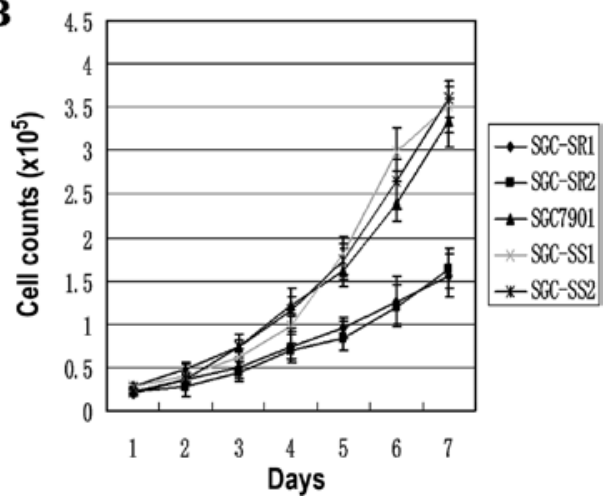

Figure 9. Growth curves of MKN-calcyclin, MKN-PC, MKN45, SGC-SR1,2, SGC-SS1,2 and untreated SGC7901 cell lines. (A) MKN-calcyclin cells grew significantly faster than MKN-PC and untreated MKN45 cells, respectively in the growth curves of MKN-calcyclin, MKN-PC and MKN45 cell lines. (B) SGC-SR1, SGC-SR2 cells grew significantly slower than SGC-SS1, SGC-SS2 and untreated SGC7901 cells.

A

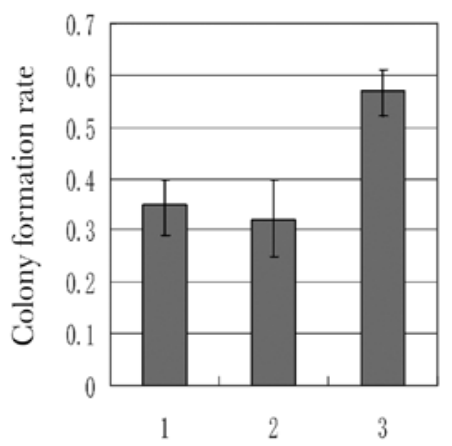

B

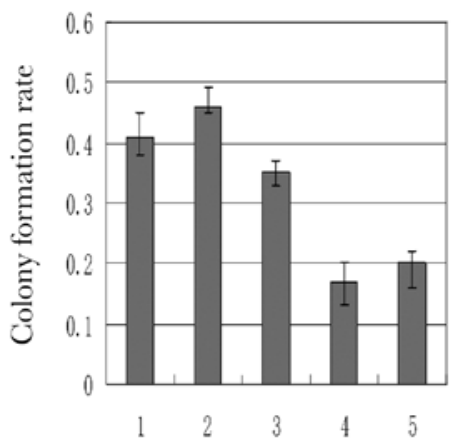

Figure 10. (A) Colony formation assay of MKN-calcyclin, MKN-PC and MKN45 cells. Colony formation rates of 1, MKN45 cells, 2, MKN-PC cells and 3 , $\mathrm{MKN}$-calcyclin cells. The results showed that the colony formation rate of $\mathrm{MKN}$-calcyclin cells was significantly higher than that of its control groups. (B) Colony formation assay of SGC-SR1, SGC-SR2, SGC-SS1, SGC-SS2 and untreated SGC7901 cell lines. Colony formation rates of 1 and 2, SGC-SS1 and SGC-SS2 cells, 3, SGC7901 cells, 4 and 5, SGC-SR1 and SGC-SR2 cells. The results showed that the colony formation rates of SGC-SR1, SGC-SR2 cell lines were significantly lower than those of their control groups, respectively.

$(\mathrm{P}<0.05)$ (Fig. 10B). It is apparent that transfection with calcyclin siRNA decreased the capacity of these tumor cells to establish colonies.

Calcyclin is important for cell cycle progression. The results of the effect of calcyclin on individual experiments measuring cell cycle progression are summarized in Tables I and II. It was clear that up-regulation or down-regulation of calcyclin gene expression led to consistent changes in the number of cells in the $\mathrm{G} 0 / \mathrm{G} 1$, S or G2/M phases. Up-regulation of calcyclin could increase the number of cells in the G2/M phase, but decreased that in the $\mathrm{S}$ phase. On the other hand, down-regulation of the calcyclin gene expression could lead to an increased number of cells in the G0/G1 phase and a decreased number of cells in the $\mathrm{S}$ phase. This appeared to be caused by specific changes in the rate at which cells passed from the G0/G1 to the $S$ phase with changes of calcyclin protein levels.

The influence of calcyclin gene on cell apoptosis. The results of the apoptosis assay showed that the average apoptosis rates of all cell clones in MKN-calcyclin, MKN-PC and untreated MKN45 groups were $6.29 \pm 0.66,2.46 \pm 0.48$ and $2.63 \pm 0.55 \%$ respectively, and there was statistically significant difference between MKN-calcyclin and their control groups $(\mathrm{P}<0.05)$. The results of the apoptosis assay in the SGC-SR1, SGC-SR2, SGC-SS1, SGC-SS2 and the untreated SGC7901 groups were $1.85 \pm 0.33,2.51 \pm 0.31,2.17 \pm 0.32,1.96 \pm 0.63$ and $2.39 \pm 0.25 \%$ respectively, and there was no statistically significant difference between them $(\mathrm{P}>0.05)$. It appeared that up-regulation but not down-regulation of calcyclin gene expression had an important influence on cell apoptosis.

The influence of calcyclin gene on cellular proliferation. Proliferating cell nuclear antigen (PCNA) reactivity patterns were widely variable, with diffuse nuclear staining. The results of the immunocytochemistry assay for PCNA showed that there were PCNA immunostained cells in the SGC-SR1, SGC-SR2, SGC-SS1, SGC-SS2 and the untreated SGC7901 groups. The brown positive signals were mainly distributed in the cell nucleus (Fig. 11A). The mean PCNA positive proportions were significantly different $(\mathrm{P}<0.05)$ between the SGC-SR1, SGC-SR2 and the control groups. As a result, the PCNA index decreased significantly with decreasing calcyclin gene expression in the SGC7901 cell, whereas the differences of the PCNA index between the control groups were not significant (Fig. 11B). 
Table I. Cell cycle distribution of the MKN-calcyclin, MKN-PC and MKN45 cells.

\begin{tabular}{lccccc}
\hline Group & Clone no. & $\mathrm{n}$ & G0/G1 $(\%)$ & G2/M $(\%)$ & $\mathrm{S}(\%)$ \\
\hline MKN-calcyclin & 8 & 3 & $42.54 \pm 5.32$ & $19.43 \pm 2.50^{\mathrm{a}}$ & $36.03 \pm 2.27^{\mathrm{a}}$ \\
MKN-PC & 13 & 3 & $44.33 \pm 3.45$ & $2.79 \pm 0.44$ & $52.88 \pm 6.43$ \\
MKN45 & 1 & 3 & $47.90 \pm 2.39$ & $3.12 \pm 5.37$ & $48.98 \pm 4.22$
\end{tabular}

${ }^{\text {a }}<0.05$ compared with the MKN45 group.

Table II. Cell cycle distribution of the SGC-SR1, SGC-SR2, SGC-SS1, SGC-SS2 and the untreated SGC7901 cells.

\begin{tabular}{lllll}
\hline Group & $\mathrm{n}$ & $\mathrm{G} 0 / \mathrm{G} 1(\%)$ & $\mathrm{G} 2 / \mathrm{M}(\%)$ & $\mathrm{S}(\%)$ \\
\hline SGC-SR1 & 3 & $53.34 \pm 4.45^{\mathrm{a}}$ & $4.45 \pm 0.40$ & $43.03 \pm 6.27^{\mathrm{a}}$ \\
SGC-SR2 & 3 & $51.03 \pm 6.25^{\mathrm{a}}$ & $7.85 \pm 2.32$ & $41.12 \pm 5.56^{\mathrm{a}}$ \\
SGC-SS1 & 3 & $35.50 \pm 5.21$ & $6.67 \pm 0.54$ & $57.83 \pm 11.67$ \\
SGC-SS2 & 3 & $42.66 \pm 4.57$ & $3.55 \pm 1.16$ & $53.79 \pm 1.34$ \\
SGC7901 & 3 & $36.22 \pm 8.43$ & $3.78 \pm 0.46$ & $60.00 \pm 7.78$ \\
\hline
\end{tabular}

${ }^{\mathrm{a}} \mathrm{P}<0.05$ compared with the SGC7901 group.

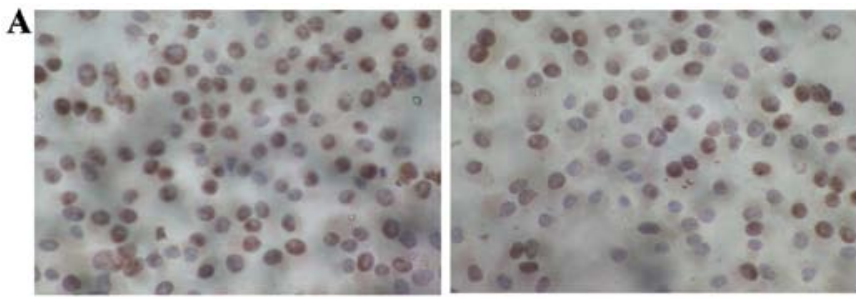

B

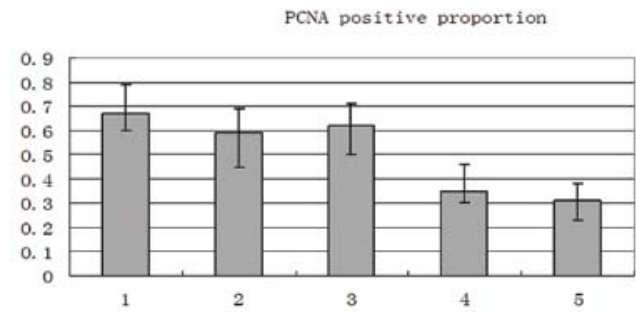

Figure 11. (A) Immunocytochemistry of PCNA in SGC-SS1 (left) and SGCSR1 (right) cells (x200). The brown positive signals were mainly distributed in the cell nucleus of SGC-SS1 and SGC-SR1 cells. The results showed that there were fewer PCNA-positive cells in SGC-SR1 than in the SGC-SS1 cells. (B) Analysis of the PCNA-positive proportion of SGC-SR1, SGC-SR2, SGCSS1, SGC-SS2 and untreated SGC7901 cells. PCNA-positive proportions of 1 and 2, SGC-SS1, and SGC-SS2 cells, 3, SGC7901 cells, 4 and 5 SGC-SR1 and SGC-SR 2 cells.

Role of calcyclin in cancer cell migration. Because individual cell migration is an important characteristic of invasive tumor cells, we examined the effects of calcyclin modulation on migration. The migration rates of MKN-calcyclin, MKN-PC, untreated MKN45 cells and calcyclin siRNA transfected cells are showed in Fig. 12. We observed that the cell migration rate was decreased when the calcyclin gene expression was up-regulated but was increased when its expression was downregulated. The statistical differences in the experimental groups and their control groups were significant $(\mathrm{P}<0.05)$.

\section{Discussion}

Upregulation of calcyclin is frequent in gastric adenocarcinoma as well as other malignancies. Although the role of calcyclin has yet to be clearly defined, it has been implicated in several cellular processes, such as cell proliferation and invasion $(37,38)$. In relation to studies performed on other tumor forms, the evaluation of calcyclin yielded conflicting results. In pancreas $(37,39)$, thyroid $(40,41)$ and colorectal $(42)$ cancers, calcyclin expression has been directly correlated with the neoplastic phenotype, being more expressed in cancer than in normal tissues. Additionally, calcyclin expression was reported to be a negative prognostic factor in pancreatic tumors (37), and in colorectal cancer it was more expressed in liver metastasis then in primary tumors (42). Completely opposite results have been obtained in osteosarcoma (43) and cholesteatoma (44), where increased calcyclin levels in tumor cells have been coupled with improved survival.

In the present study, the role of this gene in gastric cancer was investigated in vitro. Cell growth or proliferation was significantly increased in calcyclin-tansfected gastric cancer cells and significantly decreased in calcyclin-RNAi gastric cancer cells. The results of the present study provided more support for an oncogenic (proliferative) role in gastric cancer and concurred with those in pancreas, thyroid and colorectal cancers. It appears that calcyclin plays a tumor-enhancing role in adenocarcinoma, but not in other tumors such as osteosarcoma. Its role could be very different in different histological malignancies. The influence on cell growth or proliferation could be due to the direct or indirect regulation of cellular genes involved in transcription and the mitotic program as well as mediating of nuclear events associated with growth factor stimulation. In conclusion, the molecular and biological factors that control the balance between cell proliferation and 
A

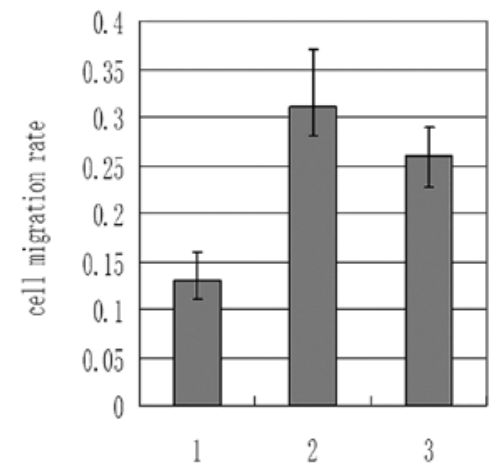

B

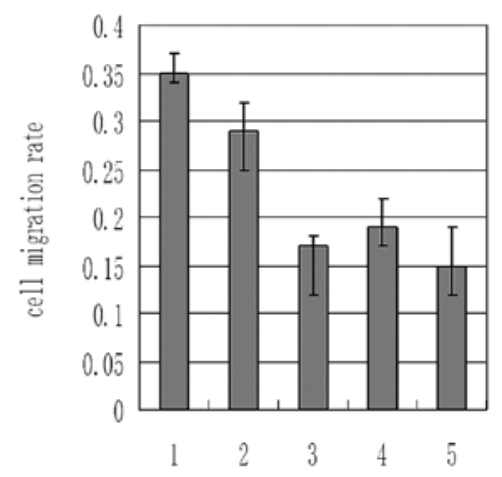

Figure 12. (A) Cell migration assay of MKN-calcyclin, MKN-PC and untreated MKN45 cell lines. Cell migration rate of 1, MKN-calcyclin cells, 2, MKN-PC cells and 3, untreated MKN45 cells. The results showed that the cell migration rate of the MKN-calcyclin cell line was significantly lower than that of its control groups. (B)Cell migration assay of SGC-SR1, SGC-SR2, SGC-SS1, SGC-SS2 and untreated SGC7901 cell lines. Cell migration rates of 1 and 2 , SGC-SR1 and SGC-SR2 cell lines 3 and 4, SGC-SS1 and SGC-SS2 cell lines and 5, the SGC7901 cell line. The results showed that the cell migration rates of the SGC-SR1, SGC-SR2 cell lines were significantly higher than those of their control groups.

apoptosis in development and progression of gastric cancers are complex, but the changes of calcyclin gene expression have an effective influence on growth and proliferation of gastric cancer cells. Research on the molecular mechanism of the influence of calcyclin on cell growth or proliferation is limited and further studies should be performed.

The results of the cell cycle assay suggest that up-regulation of calcyclin expression could increase the cells in $\mathrm{G} 2 / \mathrm{M}$ phase significantly, while down-regulation of calcyclin expression increased the proportion of cells in the G0/G1 phase. These results provide additional support for calcyclin's role in promoting cell growth and proliferation. In previous studies, calcyclin protein has been implicated in the regulation of cell growth and proliferation because its mRNA is preferentially expressed in the G1 phase of the cell cycle (45). The effects of calcyclin on cell cycling are probably mediated via interactions with various ligands including $\mathrm{Ca}^{2+} /$ phospholipid-binding proteins of the Annexin family, glyceraldehyde-3-phosphate dehydrogenase, and a $30 \mathrm{kDa}$ protein present in Ehrlich ascites tumor cells (46). It is possible that the post-translational modification of calcyclin will induce the conformational changes which direct the binding of calcyclin to a specific target, and, thereby elicit a distinct cascade of cellular events including the cell cycle. In essence, calcyclin could regulate genes involved in cell cycle regulation. To date, the complex modification of calcyclin on the cell cycle was not clear. In further research, we will investigate the molecular mechanism of the influence of calcyclin on the biological characteristics of gastric cancer cells.

Using the cell apoptosis assay, we found that up-regulation of calcyclin expression could increase the cell apoptosis rate in gastric cancer cells. It seemed that calcyclin could induce cell apoptosis, but the results of many studies have suggested that the influence of calcyclin on cell apoptosis is very complex and contradictory conclusions have been reported. Słomnicki et al (47) investigated the functional implications of the calcyclinp53 interaction by comparing various aspects of p53 activity in HEp-2 cells with either unaltered or diminished calcyclin content due to stable expression of siRNA. They found that the presence of calcyclin results in higher p53 transcriptional activity which is also reflected by higher cell susceptibility to apoptosis evoked by hydrogen peroxide. The results suggested the calcyclin could enhance cell apoptosis through the p53 pathway. Joo et al (48) demonstrated that up-regulation of calcyclin enhanced cell death and the processing of apoptosis involved the modulation of the transcriptional regulation of caspase-3. Leclerc et al (49) thought that calcyclin modulates cell apoptosis in a RAGE (receptor for advanced glycation end products)-dependent manner. Calcyclin specifically interacted with the $\mathrm{C}(1)$ and $\mathrm{C}(2)$ RAGE domains. In our research, down-regulation of calcyclin expression by specific RNAi had no detectable influence on gastric cancer cell apoptosis. The reason could be that the change of cell apoptosis had not been displayed in the short experimental time. Further research will be performed to confirm the results and identify the reason for the lack of an effect on apoptosis.

Our results showed that up-regulation or down-regulation of calcyclin expression could have a noticeable influence on cellular migration. It seemed that increased calcyclin expression could restrain cellular migration, so the gene could depress the invasion of gastric cancer cells. Otherwise, specific downregulation of the calcyclin gene enhanced cellular migration. The results are in agreement with those of Luu et al (43). They found that loss of calcyclin's expression could correlate with a metastatic phenotype. However, little is known about the exact molecular mechanism of calcyclin's impact on cellular invasion, even though it has been shown to interact with the actin cytoskeleton via tropomyosin $(38,50)$. The actin microfilament system attaches to the adherens junctions and is involved in cell contractility and adhesion-dependent signaling with the extracellular matrix (51). It is conceivable that through its interactions with the actin microfilament system, calcyclin can modulate cell adhesion, cell motility, and/or anchorageindependent growth. Interestingly, tropomyosin has been shown to suppress transformed phenotypes, such as anchorage independent growth, in both ras and src transformed cells (52-54). Therefore, the results implied that knockdown of calcyclin could not be helpful for treating the disease.

The results of the present investigation demonstrated that overexpression of calcyclin gene can influence some biological characteristics of gastric cancer cell. Calcyclin can promote 
the growth and proliferation of these cells and help tumor cells maintain the malignant phenotype. However, it can restrain the ability of invasion of gastric cancer cells. Specific knockdown of calcyclin can repress gastric cancer cell growth and proliferation, but can enhance invasion of gastric cancer cells. The gene may be a potential target for gene therapy.

\section{Acknowledgements}

The authors wish to thank Dr Gangshi Wang and nurse Weidi You and Shiping $\mathrm{Xu}$, for handling patient contacts. We wish to thank the Fourth Military Medical University of PLA for providing means for the current investigation.

\section{References}

1. Parkin DM, Bray F, Ferlay J and Pisani P: Global cancer statistics, 2002. CA Cancer J Clin 55: 74-108, 2005.

2. Crew KD and Neugut AI: Epidemiology of gastric cancer. World J Gastroenterol 12: 354-362, 2006.

3. Arber N, Shapira I, Ratan J, Stern B, Hibshoosh H, Moshkowitz M, Gammon M, Fabian I and Halpern Z: Activation of c-K-ras mutations in human gastrointestinal tumors. Gastroenterology 118: 1045-1050, 2000

4. Milne AN, Sitarz R, Carvalho R, Carneiro F and Offerhaus GJ: Early onset gastric cancer: on the road to unraveling gastric carcinogenesis. Curr Mol Med 7: 15-28, 2007.

5. Calcagno DQ, Leal MF, Seabra AD, Khayat AS, Chen ES, Demachki S, Assumpcao PP, Faria MH, Rabenhorst SH, Ferreira MV, de Arruda Cardoso Smith M and Burbano RR: Interrelationship between chromosome 8 aneuploidy, C-MYC amplification and increased expression in individuals from northern Brazil with gastric adenocarcinoma. World J Gastroenterol 12: 6207-6211, 2006.

6. Kozma L, Kiss I, Hajdu J, Szentkereszty Z, Szakall S and Ember I: $\mathrm{C}$-myc amplification and cluster analysis in human gastric carcinoma. Anticancer Res 21: 707-710, 2001.

7. Yang GF, Deng CS, Xiong YY, Gong LL, Wang BC and Luo J: Expression of nuclear factor-kappa B and target genes in gastric precancerous lesions and adenocarcinoma: association with Helicobactor pylori cagA (+) infection. World J Gastroenterol 10: 491-496, 2004

8. Tsuboi K, Hirayoshi K, Takeuchi K, Sabe H, Shimada Y, Ohshio G, Tobe $\mathrm{T}$ and Hatanaka M: Expression of the c-myc gene in human gastrointestinal malignancies. Biochem Biophys Res Commun 146: 699-704, 1987.

9. Ireland AP, Shibata DK, Chandrasoma P, Lord RV, Peters JH and DeMeester TR: Clinical significance of p53 mutations in adenocarcinoma of the esophagus and cardia. Ann Surg 231: 179-187, 2000.

10. Tahara E, Kuniyasu H, Nakayama H, Yasui W and Yokozaki H: Metastasis related genes and malignancy in human esophageal, gastric and colorectal cancers. Gan To Kagaku Ryoho 20: 326-331, 1993 (In Japanese).

11. Guo XZ, Friess H, Maurer C, Berberat P, Tang WH,Zimmermann A, Naef M, Graber HU, Korc M and Büchler MW: KAI1 is unchanged in metastatic and nonmetastatic esophageal and gastric cancers. Cancer Res 58: 753-758, 1998.

12. Schafer BW and Heizmann CW: The S100 family of EF-hand calcium-binding proteins: functions and pathology. Trends Biochem Sci 21: 134-140, 1996.

13. Lewit-Bentley A and Rety S: EF-hand calcium-binding proteins (Review). Curr Opin Struct Biol 10: 637-643, 2000.

14. Donato R: S100: a multigenic family of calcium-modulated proteins of the EF-hand type with intracellular and extracellular functional roles. Int J Biochem Cell Biol 33: 637-668, 2001.

15. Heizmann CW, Fritz G and Schafer BW: S100 proteins: structure, functions and pathology. Front Biosci 7: 1356-1368, 2002.

16. Marenholz I, Heizmann CW and Fritz G: S100 proteins in mouse and man: from evolution to function and pathology (including an update of the nomenclature). Biochem Biophys Res Commun 322: $1111-1122,2004$

17. Fano G, Biocca S, Fulle S, Mariggio MA, Belia S and Callisano P: The S-100: a protein family in search of a function. Prog Neurobiol 46: 71-82, 1995.
18. Zimmer DB, Cornwall EH, Landar A and Song W: The S100 protein family: history function, and expression. Brain Res Bull 37: 417-429, 1995.

19. Hirschhorn RR, Aller P, Yuan Z-A, Gibson CW and Baserga R: Cell-cycle-specific cDNAs from mammalian cells temperature sensitive for growth. Proc Natl Acad Sci USA 81: 6004-6008, 1984.

20. Calabretta B, Battini R, Kaczmarek L, de Riel JK and Baserga R: Molecular cloning of the cDNA for a growth factor inducible gene with strong homology to S-100, a calcium binding protein. J Biol Chem 261: 12628-12632, 1986 .

21. Kunicki $\mathrm{J}$ and Filipek A: Purification and properties of a novel $\mathrm{Ca}^{2+}$-binding protein $(10.5 \mathrm{kDa})$ from Ehrlich ascites tumour cells. Biochem J 247: 663-667, 1987.

22. Kunicki J, Filipek A, Hunziker PE, Huber S and Heizmann CW: Calcium-binding protein from mouse Ehrlich ascites tumour cells is homologous to human calcyclin. Biochem J 263: 951-956, 1989.

23. Filipek A and Kuznicki J: Calcyclin - from basic research to clinical implications. Acta Biochim Pol 40: 321-327, 1993.

24. Kuznicki J: Calcyclin. In: Guidebook to the Calcium Binding Proteins. Celio MR (ed). Sambrook and Tooze, Oxford University Press, Oxford, pp143-145, 1996.

25. Komatsu K, Andoh A, Ishiguro S, Suzuki N, Hunai H, Kobune-Fujiwara Y, Kameyama M, Miyoshi J, Akedo H and Nakamura H: Increased expression of calcyclin (calcyclin), a calcium-binding protein of the S100 family, in human colorectal adenocarcinomas. Clin Cancer Res 6: 172-177, 2000.

26. Stulík J, Osterreicher J, Koupilová K, Knízek J, Bures J, Jandík P, Langr F, Dedic K, Schäfer BW and Heizmann CW: Differential expression of the $\mathrm{Ca}^{2+}$ binding calcyclin protein in normal, preneoplastic and neoplastic colon mucosa. Eur J Cancer 36: 1050-1059, 2000.

27. Komatsu K, Murata K, Kameyama M, Ayaki M, Mukai M, Ishiguro S, Miyoshi J, Tatsuta $M$, Inoue $M$ and Nakamura $H$ : Expression of calcyclin and S100A4 in matched samples of human colorectal mucosa, primary colorectal adenocarcinomas and liver metastases. Oncology 63: 192-200, 2002.

28. Joo JH, Kim JW, Lee Y, Yoon SY, Kim JH, Paik SG and Choe IS: Involvement of NF-kappaB in the regulation of calcyclin gene expression in human hepatoblastoma cell line HepG2. Biochem Biophys Res Commun 307: 274-280, 2003.

29. Crnogorac-Jurcevic T, Missiaglia E, Blaveri E, Gangeswaran R, Jones M, Terris B, Costello E, Neoptolemos JP and Lemoine NR: Molecular alterations in pancreatic carcinoma: expression profiling shows that dysregulated expression of S100 genes is highly prevalent. J Pathol 201: 63-74, 2003.

30. Shekouh AR, Thompson CC, Prime W, Campbell F, Hamlett J, Herrington CS, Lemoine NR, Crnogorac-Jurcevic T, Buechler MW, Friess H, Neoptolemos JP, Pennington SR and Costello E: Application of laser capture microdissection combined with two-dimensional electrophoresis for the discovery of differentially regulated proteins in pancreatic ductal adenocarcinoma. Proteomics 3: 1988-2001, 2003.

31. Weterman MA, van Muijen GN, Bloemers HP and Ruiter DJ: Expression of calcyclin in human melanocytic lesions. Cancer Res 15: 6061-6066, 1993.

32. Luo X, Sharff KA, Chen J, He TC and Luu HH: Calcyclin expression and function in human osteosarcoma. Clin Orthop Relat Res 466: 2060-2070, 2008

33. Yang YQ, Zhang LJ, Dong H, Jiang CL, Zhu ZG, Wu JX, Wu YL, Han JS, Xiao HS, Gao HJ and Zhang QH: Up-regulated expression of calcyclin in human gastric cancer. J Dig Dis 8: 186-193, 2007.

34. Ning X, Sun S, Hong L, Liang J, Liu L, Han S, Liu Z, Shi Y, Li Y, Gong W, Zhang S, Chen Y, Guo X, Cheng Y, Wu K and Fan D: Calcyclin-binding protein inhibits proliferation, tumorigenicity, and invasion of gastric cancer. Mol Cancer Res 5: 1254-1262, 2007.

35. Shaobo Y, Meng wei W, Yong S, Weidi Y and Wang W: Screening differentially expressed genes of gastric adenocarcinoma by cDNA microarray. Chin J Cancer Prev Treat 11: 117-120, 2004.

36. Shao Y, Yang SB, Wang MW, Wu BY, You WD and Li H: Gene expression profile of human adenocarcinoma by cDNA microarray and clustering. Zhonghua Yi Xue Yi Chuan Xue Za Zhi 21: 110-115, 2004 (In Chinese).

37. Ohuchida K, Mizumoto K, Ishikawa N, Fujii K, Konomi H, Nagai E, Yamaguchi K, Tsuneyoshi M and Tanaka M: The role of calcyclin in pancreatic cancer development and its clinical implication as a diagnostic marker and therapeutic target. Clin Cancer Res 11: 7785-7793, 2005.

38. Breen EC and Tang K: S100A6 (calcyclin) regulates pulmonary fibroblast proliferation, morphology, and cytoskeletal organization in vitro. J Cell Biochem 88: 848-854, 2003. 
39. Vimalachandran D, Greenhalf W, Thompson C, Lüttges J, Prime W, CampbellF, Dodson A, Watson R, Crnogorac-Jurcevic T, Lemoine N, Neoptolemos J and Costello E: High nuclear S100A6 (calcyclin) is significantly associated with poor survival in pancreatic cancer patients. Cancer Res 65: 3218-3225, 2005.

40. Brown LM, Helmke SM, Hunsucker SW, Netea-Maier RT, Chiang SA, Heinz DE, Shroyer KR, Duncan MW and Haugen BR: Quantitative and qualitative differences in protein expression between papillary thyroid carcinoma and normal thyroid tissue. Mol Carcinog 45: 613-626, 2006.

41. Ito Y, Yoshida H, Tomoda C, Uruno T, Miya A, Kobayashi K, Matsuzuka F, Kakudo K, Kuma K and Miyauchi A: Expression of S100A2 and S100A6 in thyroid carcinomas. Histopathology 46: 569-575, 2005

42. Komatsu K, Kobune-Fujiwara Y, Andoh A, Ishiguro S, Hunai H, Suzuki N, Kameyama M, Murata K, Miyoshi J, Akedo H, Tatsuta M and Nakamura H: Increased expression of S100A6 at the invading fronts of the primary lesion and liver metastasis in patients with colorectal adenocarcinoma. Br J Cancer 83: 769-774, 2000.

43. Luu HH, Zhou L, Haydon RC, Deyrup AT, Montag AG, Huo P, Heck R, Heizmann CW, Peabody TD, Simon MA and He TC: Increased expression of S100A6 is associated with decreased metastasis and inhibition of cell migration and anchorage independent growth in human osteosarcoma. Cancer Lett 229: 135-148, 2005 .

44. Choufani G, Mahillon V, Decaestecker C, Lequeux T, Danguy A, Salmon I, Gabius HJ, Hassid S and Kiss R: Determination of the levels of expression of sarcolectin and calcyclin and of the percentages of apoptotic but not proliferating cells to enable distinction between recurrent and non-recurrent cholesteatomas. Laryngoscope 109: 1825-1831, 1999.

45. Calabretta B, Kaczmarek L, Mars W, Ochoa D, Gibson CW, Hirschhorn RR and Baserga R: Cell-cycle-specific genes differentially expressed in human leukemias. Proc Natl Acad Sci USA 82: 4463-4467, 1985 .
46. Zeng FY, Gerke V and Gabius HJ: Identification of Annexin II, annexin IV and glyceraldehyde-3-phosphate dehydrogenase as calcyclin-binding proteins in bovine heart. Int J Biochem 25: 1019-1025, 1998.

47. Słomnicki ŁP, Nawrot B and Leśniak W: Calcyclin binds $p 53$ and affects its activity. Int J Biochem Cell Biol 41: 784-790, 2009.

48. Joo JH, Yoon SY, Kim JH, Paik SG, Min SR, Lim JS, Choe IS, Choi I and Kim JW: S100A6 (calcyclin) enhances the sensitivity to apoptosis via the up-regulation of caspase-3 activity in Hep3B cells. J Cell Biochem 103: 1183-1197, 2008.

49. Leclerc E, Fritz G, Weibel M, Heizmann CW and Galichet A: S100B and calcyclin differentially modulate cell survival by interacting with distinct RAGE (receptor for advanced glycation end products) immunoglobulin domains. J Biol Chem 282: 3131731331, 2007.

50. Golitsina NL, Kordowska J, Wang CL and Lehrer SS: Ca2Cdependent binding of calcyclin to muscle tropomyosin. Biochem Biophys Res Commun 220: 360-365, 1996.

51. Pawlak G and Helfman DM: Cytoskeletal changes in cell transformation and tumorigenesis. Curr Opin Genet Dev 11: 41-47, 2001.

52. Prasad GL, Fuldner RA and Cooper HL: Expression of transduced tropomyosin1 cDNA suppresses neoplastic growth of cells transformed by the ras oncogene. Proc Natl Acad Sci USA 90: 7039-7043, 1993

53. Prasad GL, Masuelli L, Raj MH and Harindranath N: Suppression of src-induced transformed phenotype by expression of tropomyosin-1. Oncogene 18: 2027-2031, 1999.

54. Janssen RA and Mier JW: Tropomyosin-2 cDNA lacking the 3 untranslated region riboregulator induces growth inhibition of v-Ki-ras-transformed fibroblasts. Mol Biol Cell 8: 897-908, 1997. 